\section{OCCUPATIONAL INJURIES AMONG CHILD LABOURERS: PRELIMINARY RESULTS FROM A STUDY OF THE BRICK MANUFACTURING INDUSTRY IN FOUR COUNTRIES}

1Janessa M Graves, ${ }^{2}$ Mohammad Vaqas Ali, ${ }^{3}$ Susan E Gunn. 'College of Nursing, Washington State University, Spokane, Washington, USA; ${ }^{2}$ Institute of Social and Cultural Studies (ISCS), University of the Punjab, Lahore, Punjab, Pakistan; ${ }^{3}$ Hazardous Child Labour, International Labour Organization (ILO), Geneva, Switzerland

\subsection{6/oemed-2014-102362.166}

Objectives Internationally, brick kilns employ tens of thousands of children. Due to the extreme poverty facing families who work in this industry, child labour in this sector has been challenging to eliminate. This study assesses the association between brick kiln work and self-reported injuries among children working in brick kilns in Afghanistan, Bangladesh, Nepal, and Pakistan.

Method A mixed-methods approach developed by an international team was translated and tailored for each country. Working children (aged 11-17) were identified from 1-3 brick kiln sites. Non-working controls, matched for age, sex, and socioeconomic status were identified from nearby communities. Trained interviewers administered semi-structured questionnaires to all consenting respondents.

Results 917 working children and 788 controls participated in the study. Overall, $65.1 \%$ of cases and $29.5 \%$ of controls reported experiencing a minor cut or bruises in the last month. The estimated odds ratio (OR) of recent injury was $3.60(95 \%$ CI: 2.84-4.56) comparing working children to community controls (adjusted for age category, sex, and country). Nearly half $(48.8 \%)$ of cases and $30.5 \%$ of controls reported a "bad cut", broken bone, sprain, or burn in the last year, resulting in an adjusted OR of 2.44 (95\% CI: 1.97-3.03) comparing working children to controls.

Conclusions While the hazardous nature of brick kiln work may be evident, this study was designed to provide evidence for parents, brick kiln operators, and policy-makers who seek to remove children from this work. Additionally, this work demonstrates a model for action-oriented, occupational health and safety research in challenging environments.

\section{NOISE-INDUCED STRESS AMONG PRIMARY CARE WORKERS IN LONG TERM CARE FACILITIES IN BRITISH COLUMBIA, CANADA}

${ }^{1}$ George Astrakianakis, ${ }^{1}$ Yat Chow, ${ }^{1}$ Murray Hodgson, ${ }^{2}$ Maureen Haddock, 'Pamela Ratner. ${ }^{1}$ University of British Columbia, Vancouver, BC, Canada; ${ }^{2}$ Vancouver Coastal Health, Vancouver, BC, Canada

\subsection{6/oemed-2014-102362.167}

Objectives Sound environments in healthcare facilities are characterised and reported to be poor, and suggest adverse effects on patients and health care workers. This research aimed to examine the acoustical characteristics of long-term care facilities and evaluate the association between noise conditions and stress among residential care workers.

Method We recruited from long-term care facilities in Greater Vancouver, representing a range of building characteristics that influence background noise levels, reverberation time, and speech intelligibility index. Repeated measurements of noise (personal dosimeters and area assessments) and stress (heart rate variability (HRV) and salivary cortisol levels) were collected along with self-reported measures of stress for modelling.
Results Ninty-nine residential care workers participated, most of which were female (89\%) and registered care aides (RCAs) (58.8\%). Each participant contributed four days of measurements, on two consecutive days followed by another two consecutive days at least 2 weeks later, for a total of 392 person-days of measurements. Participants were exposed to mean A-weighted average sound pressure level of $74.8 \mathrm{dBA}$ (range: 60.3-90.2 dBA) with RCAs and evening shift workers experiencing the highest mean personal exposure levels at 75.3 and $75.8 \mathrm{dBA}$, respectively.

Licensed practical nurses (LPNs) and those working the evening shift had the highest perceived stress scores, while RCAs were found to be the most stressed group of participants, with the lowest HRV and the lowest diurnal cortisol values.

Conclusions These results suggest that noise levels experienced by residential care workers induce an autonomic stress response capable of contributing to burnout, job dissatisfaction and increased absenteeism.

\section{THE INJURY PREVENTION EFFECTS OF REGULATORY WORKPLACE SAFETY INSPECTIONS IN BRITISH COLUMBIA, CANADA FROM 2001 TO 2011}

${ }^{1}$ Kim Mcleod, ${ }^{1}$ Chris Mcleod, ${ }^{1}$ Mieke Koehoorn, ${ }^{1}$ Hugh Davies, ${ }^{2}$ Benjamin Amick. ${ }^{1}$ School of Population and Public Health, University of British Columbia, Vancouver, BC, Canada; ${ }^{2}$ Robert Stempel College of Public Health and Social Work, Florida International University, Miami, FL, USA

\subsection{6/oemed-2014-102362.168}

Objectives To determine if inspections by the workplace safety regulator (WorkSafeBC) in the Canadian Province of British Columbia are associated with a reduction in firm injury rates. Method Injury, inspection, and claims data collected by WorkSafeBC were analysed to determine the lost-time rates for all single-location firms that had been in operation in British Columbia for at least four years between the years of 2001 and 2011. Loglinear generalised estimating equations analyses were conducted to examine the effect of a workplace inspection on the change in injury rates between the year of inspection and the following year. Models were adjusted for time and sector.

Results 74510 firms met the eligibility criteria, with about 3\% of firms per year experiencing an inspection, over the time period of 2001 to 2008 . The ratio of firms inspected varied by sector, for example, about $8 \%$ of primary resources firms were inspected per year during this same period. Inspected firms had a higher injury rate (10 SLF claims per 100 FTE annually) compared to non-inspected firms (4 claims per $100 \mathrm{FTE}$ ).

Through GEE analyses, it was found that an inspection reduced injuries (beta coeff $=-0.0048(-0.0067,-0.0029))$ in the year following an inspection. The effect was greater for larger $(>=10$ FTE) (beta coeff $=-0.015(-0.021,-0.009))$ versus smaller firms $(<10$ FTE) (beta coeff $=-0.0067(-0.0094,-0.0040))$.

Conclusions These results suggest that inspections do have injury prevention effects, and the differing effects by firm characteristics may indicate the opportunity to target firms according to the most appropriate intervention.

\section{VALIDATION OF THE THAI VERSION OF A WORK- RELATED QUALITY OF LIFE SCALE}

Naesinee Chaiear. Faculty of Medicine, Khon Kaen University, Thailand

10.1136/oemed-2014-102362.169 
Objectives Our aim was (a) to assess the content validity of a Thai translation/version of a quality of work-life evaluation tool, and (b) to examine its accuracy vis-à-vis nursing in Thailand.

Method Descriptive correlation study

Forward-backward translating procedures were used to develop the Thai version of the work-related quality of life scale. Six nursing experts participated in assessing content validity and 374 registered nurses (RNs) participated in its testing. After a two-week interval, 67 of the RNs were retested. Structural validity was examined using principal components analysis and the Cronbach's alphas calculated. The respective independent sample $t$-test and intra-class correlation coefficient were used to analyse known-group validity and test-retest reliability.

Sample group: Cluster sampling was used to select 374 registered nurses from the In- and Out-patient Departments t Srinagarind Hospital, Khon Kaen University.

Results The content validity index of the scale was 0.97. Principal components analysis resulted in a seven-factor model, explaining 59\% of total variance (Cronbach's alpha for the subscales ranged between 0.65 and 0.84 , while the overall Cronbach's alpha was 0.925). The known-group validity was established in the assessment results of the difference in bureaucrats (civil servants) vs. casual employees by $\mathrm{F}(8.855,0.003)$ and t $(3.305, \mathrm{p}<0.01)$. Apparently, government employees have a better quality of work life than the university's casual employees. Good test-retest reliability was observed $(r=0.898, p<0.01)$. Conclusions The Thai version of a work-related quality of life scale appeared to be well validated and therefore useable for determining the quality of work-life among nurses in Thailand.

\section{A COHORT STUDY OF WORKERS EXPOSED TO PFOA}

Kyle Steenland, Liping Zhao, Andrea Winquist. Rollins School of Public Health, Emory U, Atlanta, Ga, USA

\subsection{6/oemed-2014-102362.170}

Objectives PFOA has been linked to several diseases, but findings are inconclusive, and there have been no incidence studies in worker populations.

Method We interviewed 3700 workers or their next-of-kin in 2009-2011 for medical history, and sought medical records to validate self-reported disease. A job-exposure matrix based on over 2000 PFOA serum measurements was used to estimate serum levels over time for each worker, while a separate estimation was made for non-occupational exposure due to drinking PFOA-contaminated water. Cumulative dose in the serum (ng/ml-years) was the metric of interest. We studied 18 disease outcomes with 15 to 1430 cases; analyses were limited to confirmed cases.

Results The median year of birth was 1951; 5\% had died. The median measured serum level was $113 \mathrm{ng} / \mathrm{ml}$ in 2005 ( $\mathrm{n}=$ 1900), compared to $4 \mathrm{ng} / \mathrm{ml}$ in the US population. Among 18 outcomes studied, only ulcerative colitis (10 year lag) showed a significant trend with increasing exposure $(\mathrm{p}=0.05)$ (RRs by quartile $1.00,3.00,3.26,6.57, \mathrm{p}$ value trend 0.05$)$, similar to earlier findings in a community cohort study in the same area. Positive but non-significant trends were also observed for prostate cancer and non-hepatitis liver disease, and female hypothyroidism, which have been implicated in other studies, No marked trends were seen for high cholesterol, which had been seen in the community study.

Conclusions Ulcerative colitis was linked to PFOA exposure among workers. Analyses of other diseases did not show marked trends. Data were limited by small numbers, a largely survivor cohort, and few low exposed referents.

\section{EXPOSURE-RESPONSE ANALYSES FOR SOLUBLE PLATINUM-SALT EXPOSED WORKERS AND SENSITISATION: A RETROSPECTIVE COHORT STUDY AMONG NEWLY EXPOSED WORKERS USING ROUTINELY COLLECTED SURVEILLANCE DATA}

${ }^{1}$ Dick Heederik, ${ }^{1}$ Jose Jacobs, 'Sadegh Samadi, 'Lutzen Portengen, ${ }^{2}$ Frits van Rooy, ${ }^{3}$ Remko Houba. ${ }^{1}$ Institute for Risk Assessment Sciences, Utrecht, The Netherlands; ${ }^{2}$ Arbo Unie, Expert Center for Chemical Risk Management, Utrecht, The Netherlands; ${ }^{3}$ Netherlands Expert Center Occupational Respiratory Diseases, Utrecht, The Netherlands

\subsection{6/oemed-2014-102362.171}

Objectives Soluble platinum salts are well known respiratory sensitising agents leading to work related sensitisation in the work environment. No quantitative exposure response relation has been described for soluble platinum salts. The objective of this study was to explore exposure response relations for soluble platinum salt exposed workers.

Method A retrospective cohort study was conducted using routinely collected health surveillance data and soluble platinum exposure data. Workers who newly entered between 1 January 2000 and 31 December 2010 were included and the relation between measured soluble platinum exposure and sensitisation (as determined by skin prick testing) was analysed in more than 1000 refinery workers from 5 refineries from whom a total of more than 1700 personal exposure measurements were available. Exposure response relations were analysed in survival analysis considering changes in exposure over time. Associations were explored for present exposure, cumulative exposure and average exposure. The exposure was lagged by $0.5,1.0,1.5$, etc. year with a maximum of 5 years.

Results A clear exposure response relation was observed, most strongly for present platinum salt exposure. Exposure lagging showed that exposure preceding sensitisation with maximally $1-$ 2 years was most strongly associated with sensitisation risk. The exposure response relationship was modified by smoking and atopy, but relative risks for smoking and atopy were only modestly elevated.

Conclusions The precision of estimate of the exposure-response relationship derived from this dataset appears superior to previous epidemiologic studies conducted on platinum salt sensitisation and may as a result have possible utility to occupational exposure standard setting.

\section{CHRONIC PROBABLE POSTTRAUMATIC STRESS DISORDER AMONG POLICE REGISTRANTS IN THE WORLD TRADE CENTRE HEALTH REGISTRY TEN YEARS AFTER 9/11/01}

${ }^{1}$ James Cone, ${ }^{2}$ Rosemarie Bowler, ${ }^{1}$ Jiehui Li, ${ }^{3}$ Erica Kornblith, ${ }^{4}$ Annum Shaikh, ${ }^{2}$ Vihra Gocheva. 'New York City Department of Health and Mental Hygiene, New York, NY, USA; ${ }^{2}$ San Francisco State University, San Francisco, CA, USA; ${ }^{3}$ California School of Professional Psychology, Alliant University, San Francisco, CA, USA; ${ }^{4}$ Emory University School of Public Health, Atlanta, GA, USA

10.1136/oemed-2014-102362.172

Objectives The World Trade Centre Health Registry (WTCHR) prospectively follows a cohort of over 71000 individuals who reported being directly exposed to the $9 / 11 / 01$ terrorist attack. 